\title{
FUZZY MULTIPLY POSITIVE IMPLICATIVE HYPER BCK-IDEALS OF HYPER BCK-ALGEBRAS
}

\author{
JIANMING ZHAN, DAJING XIANG, and ZHISONG TAN
}

Received 23 June 2003

\begin{abstract}
We consider the fuzzification of the notion of fuzzy multiply positive implicative hyper BCK-ideals of BCK-algebras and then some related results are obtained. Using the concept of level subsets, we give a characterization of a fuzzy multiply positive implicative hyper BCK-ideal. We state a relation between a fuzzy hyper BCK-ideal and a fuzzy multiply positive implicative hyper BCK-ideal. Moreover, we introduce the notions of Noetherian hyper BCKalgebras and hyper homomorphisms of hyper BCK-algebras and investigate some related properties. Finally, we introduce the concept of hyper normalization of hyper BCK-algebras and discuss related properties.
\end{abstract}

2000 Mathematics Subject Classification: 06F35, 03G25, 20N20.

1. Introduction. The hyper structure theory (also called multialgebras) was introduced in 1934 by Marty [6] at the 8th Congress of Scandinavian Mathematicians. Around the 1940s, several authors worked on hypergroups, especially in France and in the United Stated, but also in Italy, Poland, and Japan. Over the following decades, many important results appeared, but above all since the 1970s onwards the most luxuriant flourishing of hyper structures has been hyper structures with many applications to several sections of both pure and applied sciences. Jun et al. [5] applied the hyper structures to BCK-algebras, introduced the concept of a hyper BCK-algebra which is a generalization of a BCK-algebra, and investigated some related properties. In [1], Dudek and Jun obtained some properties of (maximal) normal fuzzy ideals in BCC-algebra. In [2], Dudek et al. described the properties of fuzzy BCC-ideals in BCC-algebras and its images and also gave an extension of a fuzzy BCC-ideal $\mu$ of a given BCC-subalgebra $S$ of $X$ to a fuzzy BCC-ideal $\bar{\mu}$ of $X$ such that $\mu$ and $\bar{\mu}$ have the same image. In this paper, we introduce the concept of fuzzy multiply positive implicative hyper BCK-ideals of hyper BCK-algebras and obtain some related properties.

2. Preliminaries. Let $H$ be a nonempty set endowed with a hyperoperation "॰," that is, ○ is a function from $H \times H$ to $P^{*}(H)=P(H)-\{\emptyset\}$. For two subsets $A$ and $B$ of $H$, denote by $A \circ B$ the set $\bigcup_{a \in A, b \in B} a \circ b$.

By a hyper BCK-algebra we mean a nonempty set $H$ endowed with a hyperoperation "o" and constant 0 satisfying the following axioms:

(HK1) $(x \circ z) \circ(y \circ z) \ll x \circ y$;

$(\mathrm{HK} 2)(x \circ y) \circ z=(x \circ z) \circ y$; 
(HK3) $x \circ H \ll\{x\}$;

(HK4) $x \ll y$ and $y \ll x$ imply $x=y$,

for all $x, y, z \in H$, where $x \ll y$ is denoted by $0 \in x \circ y$ and for every $A, B \subseteq H . A \ll B$ is defined by, for all $a \in A$, there exists $b \in B$ such that $a \ll b$. In this case, we call "«" the hyperorder in $H$.

In any hyper BCK-algebra $H$, the following hold:

(1) $x \circ 0 \ll\{x\}, 0 \circ x \ll\{x\}$, and $0 \circ 0 \ll\{0\}$;

(2) $(A \circ B) \circ C=(A \circ C) \circ B, A \circ B \ll A$, and $0 \circ A \ll\{0\}$;

(3) $0 \circ 0 \ll x$;

(4) $0 \ll x$;

(5) $x \circ x$;

(6) $A \ll A$;

(7) $A \subseteq B$ implies $A \ll B$;

(8) $0 \circ x=\{0\}$;

(9) $0 \circ A=\{0\}$;

(10) $A \ll\{0\}$ implies $A=\{0\}$;

(11) $x \in x \circ 0$;

(12) $x \circ 0 \ll\{y\}$ implies $x \ll y$;

(13) $y \ll z$ implies $x \circ z \ll x \circ y$;

(14) $y \circ y=\{0\}$ implies $(x \circ z) \circ(y \circ z)=\{0\}$ and $x \circ z \ll y \circ z$;

(15) $A \circ\{0\}=\{0\}$ implies $A=\{0\}$,

for all $x, y, z \in H$ and for all nonempty subsets $A, B$, and $C$ of $H$.

Proposition 2.1 [5]. In a hyper BCK-algebra, the condition (HK3) is equivalent to the condition (HK5) $x \circ y \ll\{x\}$ for all $x, y \in H$.

DEFINITION 2.2 [5]. A nonempty subset $I$ of a hyper BCK-algebra $H$ is called a hyper BCK-ideal of $H$ if

(HI1) $0 \in I$;

(HI2) $x \circ y \ll I$ and $y \in I$ imply $x \in I$, for all $x, y \in H$.

By a fuzzy set $\mu$ in $H$ we mean a function $\mu: H \rightarrow[0,1]$. For a fuzzy set $\mu$ in $H$ and $t \in[0,1]$, the set $U(\mu ; t)=\{x \in H \mid \mu(x) \geq t\}$ is called a level subset of $\mu$.

Definition 2.3 [4]. A fuzzy set $\mu$ in a hyper BCK-algebra $H$ is called a fuzzy hyper BCK-ideal of $H$ if (FHI1) $x \ll y$ implies $\mu(y) \leq \mu(x)$;

(FHI2) $\mu(x) \geq \min \left\{\inf _{a \in x \circ y} \mu(a), \mu(y)\right\}$, for all $x, y \in H$.

THEOREM 2.4 [4]. Let $\mu$ be a fuzzy set in a hyper BCK-algebra $H$. Then $\mu$ is a fuzzy hyper BCK-ideal of $H$ if and only if $U(\mu ; t)$ is a hyper BCK-ideal of $H$ whenever $U(\mu ; t) \neq \emptyset$ for all $t \in[0,1]$.

3. Multiply positive implicative hyper BCK-ideals. For any $x, y \in H, x \circ y^{n}$ denotes $(\cdots(((x \circ y) \circ y) \circ y) \circ \cdots) \circ y$ in which $y$ occurs $n$ times. 
TABLE 3.1

\begin{tabular}{c|ccc}
\hline$\circ$ & 0 & 1 & 2 \\
\hline 0 & $\{0\}$ & $\{0\}$ & $\{0\}$ \\
1 & $\{1\}$ & $\{0\}$ & $\{0\}$ \\
2 & $\{2\}$ & $\{2\}$ & $\{0,2\}$ \\
\hline
\end{tabular}

TABLE 3.2

\begin{tabular}{c|ccc}
\hline$\circ$ & 0 & 1 & 2 \\
\hline 0 & $\{0\}$ & $\{0\}$ & $\{0\}$ \\
1 & $\{1\}$ & $\{0,1\}$ & $\{0\}$ \\
2 & $\{2\}$ & $\{2\}$ & $\{0,2\}$ \\
\hline
\end{tabular}

Definition 3.1. A nonempty subset $I$ of a hyper BCK-algebra $H$ is called a multiply positive implicative hyper BCK-ideal of $H$ if it satisfies the following conditions:

(I) $0 \in I$;

(II) for every $x, y, z \in H$, there exists a natural number $k=k(x, y, z)$ such that $x \circ z^{k} \ll I$ whenever $(x \circ y) \circ z^{n} \ll I$ and $y \circ z^{m} \ll I$ for any natural numbers $m$ and $n$.

EXAMPLE 3.2. Let $H=\{0,1,2\}$. Then Table 3.1 shows a hyper BCK-algebra structure on $H$.

It is easy to check that $A=\{0,2\}$ is a multiply positive implicative hyper BCK-ideal of $H$.

Definition 3.3. Let $H$ be a hyper BCK-algebra. Then $H$ is called multiply positive implicative if, for any $x, y \in H$, there exists a positive integer $n=n(x, y)$ such that $x \circ y^{n+1}=x \circ y^{n}$.

EXAmple 3.4. Let $H=\{0,1,2\}$. Consider Table 3.2.

Then $(H, \circ, 0)$ is a multiply positive implicative hyper BCK-algebra.

THEOREM 3.5. Let $H$ be a hyper BCK-algebra, then $H$ is multiply positive if and only if, for any $x, y, z \in H$, there exists a natural number $n=n(x, y, z)$ such that $(x \circ y) \circ z^{n}=$ $\left(x \circ z^{n}\right) \circ\left(y \circ z^{n}\right)$.

Proof. Assume that for any $x, y, z \in H$, there exists a natural number $n=n(x, y, z)$ such that $(x \circ y) \circ z^{n}=\left(x \circ z^{n}\right) \circ\left(y \circ z^{n}\right)$. Putting $z=y$, then $x \circ y^{n+1}=(x \circ y) \circ y^{n}=$ $\left(x \circ y^{n}\right) \circ\left(y \circ y^{n}\right)=\left(x \circ y^{n}\right) \circ 0=x \circ y^{n}$. Hence $H$ is a multiply positive implicative hyper BCK-algebra. Conversely, let $H$ be a multiply positive implicative hyper BCKalgebra. Let $x, z \in H$, then there exists a natural number $n=n(x, z)$, such that $x \circ z^{n}=$ $x \circ z^{n+1}$, and so $x \circ z^{n}=x \circ z^{2 n}$. Hence $\left(x \circ z^{n}\right) \circ\left(y \circ z^{n}\right)=\left(x \circ z^{2 n}\right) \circ\left(y \circ z^{n}\right)=$ $\left(\left(x \circ z^{2 n-1}\right) \circ z\right) \circ\left(\left(y \circ z^{n-1}\right) \circ z\right) \ll\left(x \circ z^{2 n-1}\right) \ll \cdots \ll\left(x \circ z^{n}\right) \circ y=(x \circ y) \circ z^{n}$. On the other hand, $y \circ z^{n} \ll y$. Therefore $(x \circ y) \circ z^{n}=\left(x \circ z^{n}\right) \circ y \ll\left(x \circ z^{n}\right) \circ\left(y \circ z^{n}\right)$. Hence $(x \circ y) \circ z^{n}=\left(x \circ z^{n}\right) \circ\left(y \circ z^{n}\right)$. This completes the proof. 
TABLE 4.1

\begin{tabular}{c|ccc}
\hline$\circ$ & 0 & $a$ & $b$ \\
\hline 0 & $\{0\}$ & $\{0\}$ & $\{0\}$ \\
$a$ & $\{a\}$ & $\{0\}$ & $\{0\}$ \\
$b$ & $\{b\}$ & $\{a, b\}$ & $\{0, a, b\}$ \\
\hline
\end{tabular}

THEOREM 3.6. Let $H$ be a multiply positive implicative hyper BCK-algebra. Then the following conditions are equivalent:

(i) A is a hyper BCK-ideal;

(ii) $A$ is a multiply positive implicative hyper BCK-ideal.

Proof. (i) $\Rightarrow$ (ii). Let $x, y, z \in H$. Assume that $(x \circ y) \circ z^{n} \ll A$ and $y \circ z^{m} \ll A$. Then there exists a natural number $k=k(x, y, z)$ such that $(x \circ y) \circ z^{k}=\left(x \circ z^{k}\right) \circ\left(y \circ z^{k}\right)$. Putting $l=\max \{n, m, k\}$, then we have $(x \circ y) \circ z^{l} \ll(x \circ y) \circ z^{n} \ll A, y \circ z^{l} \ll y \circ z^{m} \ll$ $A$, and $(x \circ y) \circ z^{l}=\left(x \circ z^{l}\right) \circ\left(x \circ z^{l}\right) \circ\left(y \circ z^{l}\right)$. Since $A$ is a hyper BCK-ideal, then $x \circ z^{l} \ll A$. Hence $A$ is a multiply positive implicative hyper BCK-ideal of $H$.

(ii) $\Rightarrow$ (i). Let $x \in A, y \circ x \ll A$. Putting $z=0$, then $y \circ x=(y \circ x) \circ z \ll A, x=x \circ z \ll A$. Since $A$ is a multiply positive implicative hyper BCK-ideal, then there exists a natural number $m$, such that $y \circ z^{m} \ll A$, and thus $y \in A$. Therefore $A$ is a fuzzy hyper BCKideal of $H$.

\section{Fuzzy multiply positive implicative hyper BCK-ideals}

Definition 4.1. A fuzzy set $\mu$ in a hyper BCK-algebra $H$ is called a fuzzy multiply positive implicative hyper BCK-ideal of $H$ if it satisfies the following conditions:

(i) $x \ll y$ implies $\mu(y) \leq \mu(x)$;

(ii) for any $m, n \in \mathbb{N}$, there exists a natural number $k=k(x, y, z)$ such that

$$
\inf _{c \in x \circ z^{k}} \mu(c) \geq \min \left\{\inf _{a \in(x \circ y) \circ z^{n}} \mu(a), \inf _{b \in y \circ z^{m}} \mu(b)\right\} \quad \forall x, y, z \in H .
$$

EXAMPLE 4.2. Consider a hyper BCK-algebra $H=\{0, a, b\}$ with Cayley table (Table 4.1)

Define a fuzzy set $\mu$ in $H$ by $\mu(0)=\mu(a)=0.8$ and $\mu(b)=0.4$. It is routine to verify that $\mu$ is a fuzzy multiply positive implicative hyper BCK-ideal of $H$.

THEOREM 4.3. Let $\mu$ be a fuzzy set in a hyper BCK-algebra $H$. Then $\mu$ is a fuzzy multiply positive implicative hyper BCK-ideal of $H$ if and only if all nonempty $U(\mu ; t)$ are multiply positive implicative hyper BCK-ideals of $H$.

Proof. Let $\mu$ be a fuzzy multiply positive implicative hyper BCK-ideal of $H$ and assume $U(\mu ; t) \neq \emptyset$ for $t \in[0,1]$. Then there exists $a \in U(\mu ; t)$ and hence $\mu(a) \geq t$. Therefore $\mu(0) \geq \mu(a) \geq t$ and so $0 \in U(\mu ; t)$. Let $x, y, z \in G$ be such that $(x \circ y) \circ z^{n} \ll$ $U(\mu ; t)$ and $y \circ z^{m} \ll U(\mu ; t)$. Thus, for any $a \in(x \circ y) \circ z^{n}$ and $b \in y \circ z^{m}$, there exist $a_{0} \in U(\mu ; t)$ and $b_{0} \in U(\mu ; t)$, such that $a \ll a_{0}$ and $b \ll b_{0}$, hence $\mu\left(a_{0}\right) \leq \mu(a)$ and $\mu\left(b_{0}\right) \leq \mu(b)$. Then $\mu(a) \geq t$ and $\mu(b) \geq t$ for all $a \in(x \circ y) \circ z^{n}$ and $b \in y \circ z^{m}$. 
It follows that $\inf _{a \in(x \circ y) \circ z^{n}} \mu(a) \geq t$ and $\inf _{b \in y \circ z^{m}} \mu(b) \geq t$. By Definition 4.1, there exists a natural number $k=k(x, y, z)$ such that

$$
\inf _{c \in x \circ z^{k}} \mu(c) \geq \min \left\{\inf _{a \in(x \circ y) \circ z^{n}} \mu(a), \inf _{b \in y \circ z^{m}} \mu(b)\right\} \geq t
$$

and $x \circ z^{k} \ll U(\mu ; t)$. Therefore $U(\mu ; t)$ is a multiply positive implicative hyper BCK-ideal of $H$.

Conversely, assume that for each $t \in[0,1], U(\mu ; t)(\neq \emptyset)$ is a multiply positive implicative hyper BCK-ideal of $H$. Let $x \ll y$ and $t=\mu(y)$. Then $y \in U(\mu ; t)$ and thus $x \in U(\mu ; t)$. It follows that $\mu(x) \geq t=\mu(y)$. Let $x, y, z \in H$, and put

$$
t=\min \left\{\inf _{a \in(x \circ y) \circ z^{n}} \mu(a), \inf _{b \in y \circ z^{m}} \mu(b)\right\} .
$$

Then, for each $a \in(x \circ y) \circ z^{n}$ and $b \in y \circ z^{m}$, we have

$$
\mu(a) \geq \inf _{a_{0} \in(x \circ y) \circ z^{n}} \mu\left(a_{0}\right) \geq \min \left\{\inf _{a_{0} \in(x \circ y) \circ z^{n}} \mu\left(a_{0}\right), \inf _{b \in y_{\circ} z^{m}} \mu(b)\right\}=t .
$$

Thus $a \in U(\mu ; t)$ and so $(x \circ y) \circ z^{n} \subseteq U(\mu ; t)$. Hence $(x \circ y) \circ z^{n} \ll U(\mu ; t)$. Similarly, $y \circ z^{m} \ll U(\mu ; t)$. Since $U(\mu ; t)$ is a multiply positive implicative hyper BCK-ideal of $H$, then there exists a natural number $k=k(x, y, z)$ such that $x \circ z^{k} \ll U(\mu ; t)$, and thus

$$
\inf _{c \in x \circ z^{k}} \mu(c) \geq t=\min \left\{\inf _{a \in(x \circ y) \circ z^{n}} \mu(a), \inf _{b \in y \circ z^{m}} \mu(b)\right\} .
$$

Therefore $\mu$ is a fuzzy multiply positive implicative hyper BCK-ideal of $H$.

THEOREM 4.4. For any subset I of $H$, let $\mu_{I}$ be a fuzzy set in $H$ defined by

$$
\mu_{I}(x)= \begin{cases}t_{1} & \text { if } x \in I, \\ t_{2} & \text { otherwise }\end{cases}
$$

for all $x \in H$, where $t_{1}, t_{2} \in[0,1]$ with $t_{1}>t_{2}$. Then $I$ is a multiply positive implicative hyper BCK-ideal of $H$ if and only if $\mu_{I}$ is a fuzzy multiply positive implicative hyper $B C K$-ideal of $H$.

Proof. Assume that $I$ is a multiply positive implicative hyper BCK-ideal of $H$. Note that

$$
U\left(\mu_{I} ; t\right)= \begin{cases}\emptyset & \text { if } t_{1}<t \leq 1 \\ I & \text { if } t_{2}<t \leq t_{1} \\ H & \text { if } 0 \leq t \leq t_{2}\end{cases}
$$

which is a multiply positive implicative hyper BCK-ideal of $H$ for all $t \in[0,1]$. It follows from Theorem 4.3 that $\mu_{I}$ is a fuzzy multiply positive implicative hyper BCK-ideal of $H$. Conversely, if $\mu_{I}$ is a fuzzy multiply positive hyper BCK-ideal of $H$, then $U\left(\mu_{I} ; t\right)=I$ is a multiply positive implicative hyper BCK-ideal of $H$. 
TABLE 4.2

\begin{tabular}{c|ccc}
\hline$\circ$ & 0 & $a$ & $b$ \\
\hline 0 & $\{0\}$ & $\{0\}$ & $\{0\}$ \\
$a$ & $\{a\}$ & $\{0\}$ & $\{0\}$ \\
$b$ & $\{b\}$ & $\{a\}$ & $\{0, a\}$ \\
\hline
\end{tabular}

THEOREM 4.5. Every fuzzy multiply positive implicative hyper BCK-ideal is a fuzzy hyper BCK-ideal.

Proof. Let $\mu$ be a fuzzy multiply positive implicative hyper BCK-ideal. Then

$$
\begin{aligned}
\mu(x) & =\mu\left(x \circ 0^{k}\right) \geq \inf _{c \in x \circ 0^{k}} \mu(c) \geq \min \left\{\inf _{a \in(x \circ y) \circ 0^{n}} \mu(a), \inf _{b \in y \circ 0^{m}} \mu(b)\right\} \\
& =\min \left\{\inf _{a \in x \circ y} \mu(a), \mu(y)\right\}
\end{aligned}
$$

for all $x, y \in H$. Hence $\mu$ is a fuzzy hyper BCK-ideal of $H$.

The converse of Theorem 4.5 may not be true as seen in Cayley table (Table 4.2).

Define a fuzzy set $\mu$ in $H$ by $\mu(0)=t_{1}$ and $\mu(a)=\mu(b)=t_{2}$ for every $t_{1}>t_{2}$ in [0,1]. It can be easily checked that $\mu$ is a fuzzy multiply positive implicative hyper BCK-ideal of $H$ since

$$
\inf _{l \in b \circ a} \mu(l)=t_{2}<t_{1}=\min \left\{\inf _{m \in(b \circ a) \circ a} \mu(m), \inf _{n \in a \circ a} \mu(n)\right\}
$$

Now we give a condition for a fuzzy hyper BCK-ideal to be a fuzzy multiply positive implicative hyper BCK-ideal.

THEOREM 4.6. Let $H$ be a multiply positive implicative hyper BCK-algebra, then the following are equivalent:

(i) $\mu$ is a fuzzy hyper BCK-ideal;

(ii) $\mu$ is a fuzzy multiply positive implicative hyper BCK-ideal.

Proof. (i) $\Rightarrow($ ii). Assume that $\mu$ is a fuzzy hyper BCK-ideal of $H$. By Theorem 2.4, we have $U(\mu ; t)$ is a hyper BCK-ideal of $H$ for any $t \in[0,1]$. Thus $U(\mu ; t)$ is a multiply positive implicative hyper BCK-ideal of $H$ by Theorem 3.6. It follows from Theorem 4.3 that $\mu$ is a fuzzy multiply positive implicative hyper BCK-ideal of $H$.

(ii) $\Rightarrow$ (i). It is obtained by Theorem 4.5 .

COROLLARY 4.7. If $\mu$ is a fuzzy multiply positive implicative hyper BCK-ideal of $H$, the set $I=\{x \in H \mid \mu(x)=\mu(0)\}$ is a multiply positive implicative hyper BCK-ideal of $H$.

Proof. Clearly $0 \in I$. Let $x, y, z \in H$ be such that $(x \circ y) \circ z^{n} \ll I$ and $y \circ z^{m} \ll I$. Then for $a \in(x \circ y) \circ z^{n}$ and $b \in y \circ z^{m}$, there exist $a_{0} \in I$ and $b_{0} \in I$ such that $a \ll a_{0}$ 
and $b \ll b_{0}$. Then $\mu(a) \geq \mu\left(a_{0}\right)=\mu(0)$ and $\mu(b) \geq \mu\left(b_{0}\right)=\mu(0)$. It follows that

$$
\inf _{c \in x \circ z^{k}} \mu(c) \geq \min \left\{\inf _{a \in(x \circ y) \circ z^{n}} \mu(a), \inf _{b \in y \circ z^{m}} \mu(b)\right\}=\mu(0)
$$

and that $\mu(c)=\mu(0)$. Hence $x \circ z^{k} \ll I$, as desired.

THEOREM 4.8. If $\mu$ is a fuzzy multiply positive implicative hyper BCK-ideal of $H$, then $\mu(x)=\sup \{\alpha \in[0,1] \mid x \in U(\mu ; t)\}$ for all $x \in H$.

Proof. Let $\beta=\sup \{\alpha \in[0,1] \mid x \in U(\mu ; t)\}$ and consider an arbitrary natural number $\epsilon$. Then there exists $\alpha \in[0,1]$ such that $x \in U(\mu ; \alpha)$ and $\beta-\epsilon<\alpha$. It follows that $\mu(x) \geq \alpha>\beta-\epsilon$, so that $\mu(x) \geq \beta$ since $\epsilon$ is arbitrary. Now, let $\mu(x)=\gamma$. Then $x \in U(\mu ; \gamma)$ and so $\gamma \in\{\alpha \in[0,1] \mid x \in U(\mu ; \alpha)\}=\beta$. Therefore $\mu(x)=\beta$, as desired.

Now, we state the converse of Theorem 4.8.

THEOREM 4.9. Let $\Lambda$ be a nonempty subset of $[0,1]$ and let $\left\{I_{\alpha} \mid \alpha \in \Lambda\right\}$ be a collection of multiply positive implicative hyper BCK-ideals of $H$ such that $H=\bigcup_{\alpha \in \Lambda} I_{\alpha}$ and, for all $\alpha, \beta \in \Lambda, \beta>\alpha$ if and only if $I_{\beta} \subset I_{\alpha}$. Define a fuzzy set $\mu$ in $H$ by $\mu(x)=\sup \{\alpha \in \Lambda \mid$ $\left.x \in I_{\alpha}\right\}$ for all $x \in H$. Then $\mu$ is a fuzzy multiply positive implicative hyper BCK-ideal of $H$.

Proof. Using Theorem 4.3, it is sufficient to show that the nonempty level set $U$ ( $\mu$; $\alpha$ ) of $\mu$ is a multiply positive implicative hyper BCK-ideal of $H$ for every $\delta \in[0,1]$. We should consider two cases as follows:

(i) $\delta=\sup \{\alpha \in \Lambda \mid \alpha<\delta\}$,

(ii) $\delta \neq \sup \{\alpha \in \Lambda \mid \alpha<\delta\}$.

For the case (i), we have $x \in U(\mu ; \delta) \Leftrightarrow x \in I_{\alpha}$ for all $\alpha<\delta \Leftrightarrow x \in \bigcap_{\alpha<\delta} I_{\alpha}$ whence $U(\mu ; \delta)=\bigcap_{\alpha<\delta} I_{\alpha}$, which is a multiply positive implicative hyper BCK-ideal of $H$. Case (ii) implies that there exists $\epsilon>0$ such that $(\delta-\epsilon, \delta) \cap \Lambda=\emptyset$. If $x \in \bigcup_{\alpha \geq \delta} I_{\alpha}$, then $x \in I_{\alpha}$ for some $\alpha \geq \delta$. It follows that $\mu(x) \geq \alpha \geq \delta$, so that $x \in U(\mu ; \delta)$. This proves that $\bigcup_{\alpha \geq \delta} I_{\alpha} \subseteq U(\mu ; \delta)$. Assume that $x \notin \bigcup_{\alpha \geq \delta} I_{\alpha}$. Then $x \notin I_{\alpha}$ for all $\alpha \geq \delta$, which implies that $x \notin I_{\alpha}$ for all $\alpha>\delta-\epsilon$, that is, if $x \in I_{\alpha}$, then $\alpha \leq \delta-\epsilon$. Thus $\mu(x) \leq \delta-\epsilon<\delta$ and so $x \notin U(\mu ; \delta)$. Therefore $U(\mu ; \delta) \subseteq \bigcup_{\alpha \geq \delta} I_{\alpha}$, and consequently $U(\mu ; \alpha)=\bigcup_{\alpha \geq \delta} I_{\alpha}$, which is a multiply positive implicative hyper BCK-ideal of $H$.

\section{Noetherian hyper BCK-algebras}

DEFINITION 5.1. A hyper BCK-algebra $H$ is said to satisfy the ascending (resp., descending) chain condition (briefly, ACC (resp., DCC)) if, for every ascending (resp., descending) sequence $A_{1} \subseteq A_{2} \subseteq \cdots$ (resp., $A_{1} \supseteq A_{2} \supseteq \cdots$ ) of multiply positive implicative hyper BCK-ideals of $H$, there exists a natural number $n$ such that $A_{n}=A_{k}$ for all $n \geq k$.

DEFINITION 5.2. A hyper BCK-algebra $H$ is said to be Noetherian if $H$ satisfies the ACC for multiply positive implicative hyper BCK-ideals. 
Let $\mu$ be a fuzzy set in $H$. We note that $\operatorname{Im} \mu$ is a bounded subset of $[0,1]$. Hence, we can consider a sequence of elements of $\operatorname{Im} \mu$ which is either increasing or decreasing.

THEOREM 5.3. Let $H$ be a hyper BCK-algebra satisfying DCC and let $\mu$ be a fuzzy multiply positive implicative hyper BCK-ideals of $H$. If a sequence of elements of $\operatorname{Im} \mu$ is strictly increasing, then $\mu$ has a finite number of values.

Proof. Let $\left\{t_{n}\right\}$ be a strictly increasing sequence of elements of $\operatorname{Im} \mu$. Then $0 \leq t_{1}<$ $t_{2}<\cdots \leq 1$. Define $\mu_{r}=\left\{x \in H \mid \mu(x) \geq t_{r}\right\}, r=1,2,3, \ldots$. Then $\mu_{r}$ is a multiply positive implicative hyper BCK-ideal by Theorem 4.3. Let $x \in \mu_{r}$, then $\mu(x) \geq t_{r}>t_{r-1}$, which implies that $x \in \mu_{r-1}$. Hence $\mu_{r} \subseteq \mu_{r-1}$. Since $t_{r-1} \in \operatorname{Im} \mu$, there exists $x_{r-1} \in$ $H$, such that $\mu\left(x_{r-1}\right)=t_{r-1}$. It follows that $x_{r-1} \in \mu_{r-1}$, but $x_{r-1} \notin \mu_{r}$. Thus $\mu_{r} \subsetneq$ $\mu_{r-1}$, and so we obtain a strictly descending sequence $\mu_{1} \supsetneq \mu_{2} \supsetneq \mu_{3} \supsetneq \cdots$ of multiply positive implicative hyper BCK-ideals of $H$ which is not terminating. This contradicts the assumption that $H$ satisfies DCC, completing the proof.

Now we consider the converse of Theorem 5.3.

THEOREM 5.4. Let $H$ be a hyper BCK-algebra. If every fuzzy multiply positive implicative hyper BCK-ideal of $H$ has a finite number of values, then $H$ satisfies DCC.

Proof. Suppose $H$ does not satisfy DCC, then there exists a strictly descending chain $A_{0} \supsetneq A_{1} \supsetneq A_{2} \supsetneq \cdots$ of multiply positive implicative hyper BCK-ideals of $H$. Define a fuzzy set $v$ in $H$ by

$$
v(x)= \begin{cases}\frac{i}{i+1} & \text { if } x \in A_{i}-A_{i-1}, i=0,1,2, \ldots, \\ 1 & \text { if } x \in \bigcap_{i=0}^{\infty} A_{i}\end{cases}
$$

where $A_{0}$ stands for $H$. We prove that $v$ is a fuzzy multiply positive implicative hyper BCK-ideal of $H$. Let $x, y \in H$ be such that $x \ll y$. If $y \in \bigcup_{i=0}^{\infty} A_{i}$, then $x \in \bigcup_{i=0}^{\infty} A_{i}$, and thus $v(x)=1=v(y)$. If $y \in A_{i}-A_{i-1}$ for $i=0,1,2, \ldots$, then $x \in A_{i}$, and thus $v(x) \geq i /(i+1)=v(y)$. Let $x, y, z \in H$. Assume that $(x \circ y) \circ z^{n} \ll A_{i}-A_{i=1}$ and $y \circ z^{m} \in A_{j}-A_{j+1}$ for $i=0,1,2, \ldots, j=0,1,2, \ldots$. Without loss of generality, we may assume that $i \leq j$. Then clearly $y \circ z^{m} \ll A_{i}$. Since $A_{i}$ is a multiply positive implicative hyper BCK-ideal, then there exists a natural number $k=k(x, y, z)$ such that $x \circ z^{k} \ll A_{i}$. Hence

$$
\inf _{c \in x \circ z^{k}} v(c) \geq \frac{i}{i+1}=\min \left\{\inf _{a \in(x \circ y) \circ z^{n}} v(a), \inf _{b \in y \circ z^{m}} v(b)\right\}
$$

If $(x \circ y) \circ z^{n} \ll \bigcap_{i=0}^{\infty} A_{i}$ and $y \circ z^{m} \ll \bigcap_{i=0}^{\infty} A_{i}$, then there exists a natural number $k=k(x, y, z)$ such that $x \circ z^{k} \ll \bigcap_{i=0}^{\infty} A_{i}$. Thus

$$
\inf _{c \in x \circ z^{k}} v(c)=1=\min \left\{\inf _{a \in(x \circ y) \circ z^{n}} v(a), \inf _{b \in y \circ z^{m}} v(b)\right\}
$$


If $(x \circ y) \circ z^{n}$ does not belong to $\bigcap_{i=0}^{\infty} A_{n}$ and $y \circ z^{m} \ll \bigcap_{i=0}^{\infty} A_{i}$, then there exists $t \in \mathbb{N}$ such that $(x \circ y) \circ z^{k} \ll A_{t}-A_{t+1}$. It follows that $x \circ z^{k} \ll A_{t}$ so that

$$
\inf _{c \in x \circ z^{k}} v(x) \geq \frac{t}{t+1}=\min \left\{\inf _{a \in(x \circ y) \circ z^{n}} v(a), \inf _{b \in y \circ z^{m}} v(b)\right\} .
$$

Finally assume that $(x \circ y) \circ z^{n} \ll \bigcap_{i=0}^{\infty} A_{i}$ and $y \circ z^{m}$ does not belong to $\bigcap_{i=0}^{\infty} A_{i}$, then $y \circ z^{k} \in A_{r}-A_{r+1}$ for some $r \in \mathbb{N}$. Hence there exists a natural number $k=k(x, y, z)$ such that $x \circ z^{k} \ll A_{r}$, and thus

$$
\inf _{c \in x \circ z^{k}} v(c) \geq \frac{r}{r+1}=\min \left\{\inf _{a \in(x \circ y) \circ z^{n}} v(a), \inf _{b \in y \circ z^{m}} v(b)\right\} .
$$

Consequently we find that $v$ is a fuzzy multiply positive implicative hyper BCK-ideal and $v$ has an infinite number of different values. This is a contradiction and the proof is complete.

THEOREM 5.5. For any hyper BCK-algebra $H$, the following are equivalent:

(i) $H$ is Noetherian;

(ii) the set of values of any fuzzy multiply positive hyper BCK-ideal on $H$ is a wellordered subset of $[0,1]$.

Proof. (i) $\Rightarrow$ (ii). Suppose that $\mu$ is a fuzzy multiply positive implicative hyper BCKideal whose set of values is not a well-ordered subset of $[0,1]$. Then there exists a strictly decreasing sequence $\left\{t_{n}\right\}$ such that $\mu\left(x_{n}\right)=t_{n}$. Let $B_{n}=\left\{x \in H \mid \mu(x) \geq t_{n}\right\}$, then $B_{1} \subsetneq B_{2} \subsetneq \cdots$ is a strictly ascending chain of multiply positive implicative hyper BCK-ideals of $H$, contradicting the assumption that $H$ is Noetherian.

(ii) $\Rightarrow$ (i). Assume that condition (ii) is satisfied and $H$ is not Noetherian. Then there exists a strictly ascending chain

$$
A_{1} \subsetneq A_{2} \subsetneq A_{3} \subsetneq \cdots
$$

of multiply positive implicative hyper BCK-ideals of $H$. Suppose $A=\bigcup_{r \in \mathbb{N}}^{A_{r}}$. Then $A$ is a multiply positive implicative hyper BCK-ideal of $H$. Define a fuzzy set $v$ in $H$ by

$$
v(x)= \begin{cases}0 & \text { if } x \notin A_{r}, \\ \frac{1}{r} & r=\min \left\{n \in \mathbb{N} \mid x \in A_{r}\right\} .\end{cases}
$$

We claim that $v$ is a fuzzy multiply positive implicative hyper BCK-ideal of $H$. Let $x, y \in$ $H$ be such that $x \ll y$. If $y \in A_{r}-A_{r-1}$ for $r=2,3, \ldots$, then $x \in A_{r}$. Hence $v(x) \geq$ $1 / r=v(y)$. If $y \in A_{r}$, then $x \in A_{r}$. Hence $v(x) \geq 1 / r \geq v(y)$. Now let $x, y, z \in H$. If $(x \circ y) \circ z^{n} \ll A_{r}-A_{r-1}$ and $y \ll A_{r}-A_{r-1}$ for $r=2,3, \ldots$, then there exists a natural number $k=k(x, y, z)$ such that $x \circ z^{k} \ll A_{r}$. It follows that

$$
\inf _{c \in x \circ z^{k}} v(c) \geq \frac{1}{r}=\min \left\{\inf _{a \in(x \circ y) \circ z^{n}} v(a), \inf _{b \in y \circ z^{m}} v(b)\right\} .
$$

Assume that $(x \circ y) \circ z^{n} \ll A_{r}$ and $y \circ z^{m} \in A_{r}-A_{s}$ for $s<r$. Since $A_{r}$ is a multiply positive implicative hyper BCK-ideal, then there exists a natural number $k=k(x, y, z)$ 
such that $x \circ z^{k} \in A_{r}$. Hence

$$
\inf _{c \in x \circ z^{k}} v(c) \geq \frac{1}{r} \geq \frac{1}{s+1} \geq \inf _{b \in y_{\circ} z^{m}} v(b)
$$

and so

$$
\inf _{c \in x \circ z^{k}} v(c) \geq \min \left\{\inf _{a \in(x \circ y) \circ z^{n}} v(a), \inf _{b \in y \circ z^{m}} v(b)\right\} .
$$

Similarly for the case $(x \circ y) \circ z^{n} \ll A_{r}-A_{s}$ and $y \circ z^{m} \ll A_{r}$, there exists a natural number $k=k(x, y, z)$ such that (5.10) holds: thus $v$ is a fuzzy multiply positive implicative hyper BCK-ideal of $H$. Since chain (5.6) is not terminating, $v$ has a strictly descending sequence of values. This contradicts the assumption that the value set of any fuzzy multiply positive implicative hyper BCK-ideal is well ordered. Hence $H$ is Noetherian.

We note that a set is well ordered if and only if it does not contain any infinite descending sequence.

THEOREM 5.6. Let $S=\left\{t_{1}, t_{2}, \ldots\right\} \bigcup\{0\}$, where $\left\{t_{n}\right\}$ is a strictly decreasing sequence $(0,1)$. Then a hyper BCK-algebra is Noetherian if and only if, for each fuzzy multiply positive implicative hyper BCK-ideal of $H, \operatorname{Im} \mu \subseteq S$ implies that there exists a positive integer $r_{0}$ such that $\operatorname{Im} \mu \subseteq\left\{t_{1}, t_{2}, \ldots, t_{r_{0}}\right\} \bigcup\{0\}$.

Proof. Assume that $H$ is a Noetherian hyper BCK-algebra and let $\mu$ be an ideal of $H$. Then by Theorem $5.5, \operatorname{Im} \mu$ is a well-ordered subset of $[0,1]$, and so the condition is necessary.

Conversely, assume that the condition is satisfied. Suppose $H$ is not Noetherian. Then there exists a strictly ascending chain of multiply positive implicative hyper BCK-ideals

$$
A_{1} \subsetneq A_{2} \subsetneq A_{3} \subsetneq \cdots
$$

Define a fuzzy set $\mu$ in $H$ by

$$
\mu(x)= \begin{cases}t_{1} & \text { if } x \in A_{1}, \\ t_{r} & \text { if } x \in A_{r}-A_{r-1}, r=2,3, \ldots, \\ 0 & \text { if } x \in H-\bigcup_{r=1}^{\infty} A_{r} .\end{cases}
$$

Let $x, y \in H$ be such that $x \ll y$. If $y \in H-\bigcup_{r=1}^{\infty} A_{r}$, then $x \in H-\bigcup_{r=1}^{\infty} A_{r}$, and thus $\mu(x)=0=\mu(y)$. If $y \in A_{r}-A_{r-1}$, then $x \in A_{r}$, and thus $\mu(x) \geq t_{r}=\mu(y)$. If $y \in A_{1}$, then $x \in A_{1}$, and thus $\mu(x)=t_{1}=\mu(y)$. Now let $x, y, z \in H$. If either $(x \circ y) \circ z^{n} \ll$

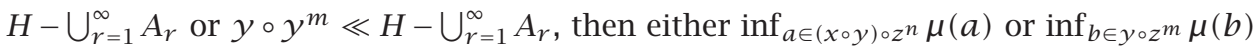
is equal to 0 and hence

$$
\inf _{c \in x \circ z^{k}} \mu(c) \geq \min \left\{\inf _{a \in(x \circ y) \circ z^{n}} \mu(a), \inf _{b \in y \circ z^{m}} \mu(b)\right\} .
$$


If $(x \circ y) \circ z^{n} \ll A_{1}$ and $y \circ z^{m} \ll A_{1}$, then there exists a natural number $k=k(x, y, z)$ such that $x \circ z^{k} \ll A_{1}$, and thus

$$
\inf _{c \in x \circ z^{k}} \mu(c)=t_{1}=\min \left\{\inf _{a \in(x \circ y) \circ z^{n}} \mu(a), \inf _{b \in y \circ z^{m}} \mu(b)\right\} .
$$

If $(x \circ y) \circ z^{n} \ll A_{r}-A_{r-1}$ and $y \circ z^{m} \in A_{r}-A_{r-1}$, then there exists a natural number $k=k(x, y, z)$ such that $x \circ z^{k} \ll A_{r}$. Hence

$$
\inf _{c \in x \circ z^{k}} \mu(c) \geq t_{r}=\min \left\{\inf _{a \in(x \circ y) \circ z^{n}} \mu(a), \inf _{b \in y \circ z^{m}} \mu(b)\right\} .
$$

Assume that $(x \circ y) \circ z^{n} \ll A_{1}$ and $y \circ z^{m} \in A_{r}-A_{r-1}$ for $r=2,3, \ldots$. Then there exists a natural number $k=k(x, y, z)$ such that $x \circ z^{k} \ll A_{r}$, and therefore

$$
\inf _{c \in x \circ z^{k}} \mu(c) \geq t_{r}=\min \left\{t_{1}, t_{2}\right\}=\min \left\{\inf _{a \in(x \circ y) \circ z^{n}} \mu(a), \inf _{b \in y \circ z^{m}} \mu(b)\right\} .
$$

Similarly, for $(x \circ y) \circ z^{n} \ll A_{r}-A_{r-1}$ and $y \circ z^{m} \ll A_{1}, r=2,3, \ldots$, we obtain

$$
\inf _{c \in x \circ z^{k}} \mu(c) \geq t_{r}=\min \left\{\inf _{a \in(x \circ y) \circ z^{n}} \mu(a), \inf _{b \in y \circ z^{m}} \mu(b)\right\} .
$$

Consequently, $\mu$ is a fuzzy multiply positive implicative hyper BCK-ideal. This contradicts our assumption. Hence $H$ is Noetherian.

\section{Hyper homomorphism of hyper BCK-algebras}

DEFINITION 6.1 [3]. A mapping $f: G \rightarrow H$ of hyper BCK-algebras is called a hyper homomorphism if

(I) $f(0)=0$;

(II) $f(x \circ y)=f(x) \circ f(y)$ for all $x, y \in G$.

LEMMA 6.2 [3]. Let $f: G \rightarrow H$ be a hyper homomorphism of hyper BCK-algebras. If $x \ll y$ in $G$, then $f(x) \ll f(y)$ in $H$.

THEOREM 6.3. Let $f: G \rightarrow H$ be a hyper homomorphism of hyper BCK-algebras. If I is a multiply positive implicative hyper BCK-ideal of $H$, then $f^{-1}(I)$ is a multiply positive implicative hyper BCK-ideal of $G$.

Proof. Clearly, $0 \in f^{-1}(I)$. Let $x, y, z \in G$ be such that $(x \circ y) \circ z^{n} \ll f^{-1}(I)$ and $y \circ z^{m} \ll f^{-1}(I)$. Then for every $a \in(x \circ y) \circ z^{n}$ and $b \in y \circ z^{m}$, there exist $a_{0} \in f^{-1}(I)$ and $b_{0} \in f^{-1}(I)$ such that $a \ll a_{0}$ and $b \ll b_{0}$, that is, $0 \in a \circ a_{0}$ and $0 \in b \circ b_{0}$. It follows that

$$
\begin{gathered}
0=f(0) \in f\left(a \circ a_{0}\right)=f(a) \circ f\left(a_{0}\right) \subseteq f\left((x \circ y) \circ z^{n}\right) \circ I=\left((f(x) \circ f(y)) \circ f(z)^{n}\right) \circ I, \\
0=f(0) \in f\left(b \circ b_{0}\right)=f(b) \circ f\left(b_{0}\right) \subseteq f\left(y \circ z^{m}\right) \circ I=\left(f(y) \circ f(z)^{m}\right) \circ I .
\end{gathered}
$$


Hence $(f(x) \circ f(y)) \circ f(z)^{n} \ll I$ and $f(y) \circ f(z)^{m} \ll I$. Since $I$ is a multiply positive implicative hyper BCK-ideal of $H$, then there exists a natural number $k=k(x, y, z)$ such that $f(x) \circ f(z)^{k} \ll I$, that is, $f\left(x \circ z^{k}\right) \ll I$, so that $x \circ z^{k} \ll f^{-1}(I)$. Hence $f^{-1}(I)$ is a multiply positive implicative hyper BCK-ideal of $G$.

Now we put forward the following open question.

QUESTION 1. Is it true of the converse of Theorem 6.3?

THEOREM 6.4. Let $f: G \rightarrow H$ be an onto hyper homomorphism of hyperBCK-algebras. If $v$ is a fuzzy multiply positive implicative hyper BCK-ideal of $H$, then the hyper homomorphic preimage $\mu$ of $v$ under $f$, that is, the fuzzy set $\mu$ in $G$ defined by $\mu(x)=v(f(x))$ for all $x \in G$, is a fuzzy multiply positive implicative hyper BCK-ideal of $G$.

Proof. Let $x, y \in G$ be such that $x \ll y$. Then $0 \in x \circ y$, and so $0=f(0) \in f(x \circ$ $y)=f(x) \circ f(y)$. Therefore, $f(x) \ll f(y)$ in $H$. Thus $\mu(x)=v(f(x)) \geq v(f(y))=$ $\mu(y)$ for any $x, y, z \in G$, then

$$
\begin{aligned}
\inf _{c \in x \circ z^{k}} \mu(c) & \geq \inf _{f(c) \in f\left(x \circ z^{k}\right)} v(f(c)) \\
& \geq \min \left\{\inf _{f(a) \in f\left((x \circ y) \circ z^{n}\right)} v(f(a)), \inf _{f(b) \in f\left(y \circ z^{m}\right)} v(f(b))\right\} \\
& =\min \left\{\inf _{a \in(x \circ y) \circ z^{n}} \mu(a), \inf _{b \in y \circ z^{m}} \mu(b)\right\}
\end{aligned}
$$

for all $a, b, c \in H$. Therefore $\mu$ is a fuzzy multiply positive implicative hyper BCK-ideal of $G$.

Now we put forward another open question.

QUESTION 2. Is it true of the converse of Theorem 6.4?

\section{Hyper normalization of hyper BCK-algebras}

Definition 7.1. A fuzzy multiply positive implicative hyper BCK-ideal $\mu$ of $H$ is said to be hypernormal if there exists $x \in H$ such that $\mu(x)=1$.

EXAMPLE 7.2. Let $H=\{0, a, b\}$ in which the hyperoperation " 0 " is given by Table 4.1.

Then $H$ is a hyper BCK-algebra. Define $\mu: H \rightarrow[0,1]$ by $\mu(0)=\mu(a)=1, \mu(b)=0.5$ is a hypernormal fuzzy multiply positive implicative hyper BCK-ideal of $H$.

Note that if $\mu$ is a hypernormal fuzzy multiply positive implicative hyper BCK-ideal of $H$, then $\mu(0)=1$, and hence $\mu$ is hypernormal if and only if $\mu(0)=1$.

THEOREM 7.3. Given a fuzzy multiply positive implicative hyper BCK-ideal of $H$, let $\mu^{+}$be a fuzzy set in $H$, defined by $\mu^{+}(x)=\mu(x)+1-\mu(0)$ for all $x \in H$. Then $\mu^{+}$is $a$ hypernormal fuzzy multiply positive implicative hyper BCK-ideal of $H$ which contains $\mu$, that is, $\mu^{+}(x) \geq \mu(x)$ for all $x \in H$. 
Proof. Let $x, y \in H$ be such that $x \ll y$, then $\mu^{+}(x)=\mu(x)+1-\mu(0) \geq \mu(y)+1-$ $\mu(0)=\mu^{+}(y)$. For any $x, y, z \in H$, we have

$$
\begin{aligned}
\inf _{c \in x \circ z^{k}} \mu^{+}(c) & =\inf _{c \in x \circ z^{k}}(\mu(c)+1-\mu(0))=1-\mu(0)+\inf _{c \in z^{k}} \mu(c) \\
& \geq 1-\mu(0)+\min \left\{\inf _{a \in(x \circ y) \circ z^{k}} \mu(a), \inf _{b \in y \circ z^{m}} \mu(b)\right\} \\
& =\min \left\{\inf _{a \in(x \circ y) \circ z^{n}} \mu(a)+1-\mu(0), \inf _{b \in y \circ z^{m}} \mu(b)+1-\mu(0)\right\} \\
& =\min \left\{\inf _{a \in(x \circ y) \circ z^{n}} \mu^{+}(a), \inf _{b \in y \circ z^{m}} \mu^{+}(b)\right\} .
\end{aligned}
$$

Hence $\mu^{+}$is a hypernormal fuzzy multiply positive implicative hyper BCK-ideal, and obviously $\mu \subseteq \mu^{+}$.

THEOREM 7.4. Let $\mu$ be a fuzzy multiply positive implicative hyper BCK-ideal of $H$ and let $\mu^{*}$ be a fuzzy set in $H$ defined by $\mu^{*}(x)=(1 / \mu(0)) \mu(x)$ for all $x \in H$, where $\mu(0) \neq 0$. Then $\mu^{*}$ is a hypernormal fuzzy multiply positive implicative hyper BCK-ideal of $H$ containing $\mu$.

Proof. Let $x, y \in H$ be such that $x \ll y$, then $\mu(x) \geq \mu(y)$, and thus $\mu^{*}(x)=$ $(1 / \mu(0)) \mu(x) \geq(1 / \mu(0)) \mu(y)=\mu^{*}(y)$. Now for $x, y, z \in H$, we have

$$
\begin{aligned}
\inf _{c \in x \circ z^{k}} \mu^{*}(c) & =\inf _{c \in x \circ z^{k}} \frac{1}{\mu(0)} \mu(c) \\
& =\frac{1}{\mu(0)} \inf _{c \in x \circ y^{k}} \mu(c) \geq \frac{1}{\mu(0)} \min \left\{\inf _{a \in(x \circ y) \circ z^{n}} \mu(a), \inf _{b \in y \circ z^{m}} \mu(b)\right\} \\
& =\min \left\{\frac{1}{\mu(0)} \inf _{a \in(x \circ y) \circ z^{n}} \mu(a), \frac{1}{\mu(0)} \inf _{b \in y \circ z^{m}} \mu(b)\right\} \\
& =\min \left\{\inf _{a \in(x \circ y) \circ z^{n}} \mu^{*}(a), \inf _{b \in y \circ z^{m}} \mu^{*}(b)\right\}
\end{aligned}
$$

and $\mu^{*}(x)=(1 / \mu(0)) \mu(x) \geq \mu(x)$ for all $x \in H$. This completes the proof.

COROLlaRY 7.5. If $\mu$ is a fuzzy multiply positive implicative hyper BCK-ideal of $H$ satisfying $\mu^{*}(x)=0$ for some $x \in H$, then $\mu(x)=0$.

COROLLARY 7.6. A fuzzy multiply positive implicative hyper BCK-ideal $\mu$ of $H$ is hypernormal if and only if $\mu^{*}=\mu$.

COROLLARY 7.7. If $\mu$ is a fuzzy multiply positive implicative hyper BCK-ideal of $H$, then $\left(\mu^{*}\right)^{*}=\mu^{*}$.

COROLLARY 7.8. If $\mu$ is a hypernormal fuzzy multiply positive implicative hyper BCKideal of $H$, then $\left(\mu^{*}\right)^{*}=\mu$. 
THEOREM 7.9. Let $\mu$ be a nonconstant hypernormal multiply positive implicative hyper BCK-ideal of $H$, which is maximal in the poset of hypernormal fuzzy multiply positive implicative hyper BCK-ideals under inclusion. Then $\mu$ takes only the values 0 and 1.

Proof. Since $\mu$ is hypernormal, we have $\mu(0)=1$. Let $x \in H$ be such that $\mu(x) \neq 1$. It is sufficient to show that $\mu(x)=0$. If not, then there exists $w \in H$ such that $0<\mu(w)<$ 1. Define a fuzzy set $v$ in $H$ by $v(x)=(1 / 2)(\mu(x)+\mu(w))$ for all $x \in H$. Clearly, $v$ is well defined. Let $x, y \in H$ be such that $x \ll y$, then $\mu(x) \geq \mu(y)$, and thus

$$
v(x)=\frac{1}{2}(\mu(x)+\mu(w)) \geq \frac{1}{2}(\mu(y)+\mu(w))=v(y) .
$$

For any $x, y, z \in H$, we have

$$
\begin{aligned}
\inf _{c \in x \circ z^{k}} v(c) & =\inf _{c \in x \circ z^{k}} \frac{1}{2}(\mu(c)+\mu(w))=\frac{1}{2}\left(\inf _{c \in x \circ z^{k}} \mu(c)+\mu(w)\right) \\
& \geq \frac{1}{2}\left(\min \left\{\inf _{a \in(x \circ y) \circ z^{n}} \mu(a), \inf _{b \in y \circ z^{m}} \mu(b)\right\}+\mu(w)\right) \\
& =\min \left\{\frac{1}{2}\left(\inf _{a \in(x \circ y) \circ z^{n}} \mu(a)+\mu(w)\right), \frac{1}{2}\left(\inf _{b \in y \circ z^{m}} \mu(b)+\mu(w)\right)\right\} \\
& =\min \left\{\inf _{a \in(x \circ y) \circ z^{n}} v(x), \inf _{b \in y \circ z^{m}} v(w)\right\} .
\end{aligned}
$$

Hence $v$ is a fuzzy multiply positive implicative hyper BCK-ideal of $H$. By Theorem 7.3, $v^{+}$is a hypernormal fuzzy multiply positive hyper BCK-ideal of $H$, where $v^{+}$is defined by $v^{+}(x)=v(x)+1-v(0)$ for all $x \in H$. Note that

$$
\begin{aligned}
v^{+}(w) & =v(w)+1-v(0)=\frac{1}{2}(\mu(w)+\mu(w))+1-\frac{1}{2}(\mu(0)+\mu(0)) \\
& =\frac{1}{2}(\mu(w)+1)>\mu(w)
\end{aligned}
$$

and $v^{+}(w)<1=v^{+}(0)$. It follows that $v^{+}$is nonconstant and $\mu$ is not maximal. This is a contradiction, ending the proof.

\section{REFERENCES}

[1] W. A. Dudek and Y. B. Jun, Normalizations of fuzzy BCC-ideals in BCC-algebras, Math. Morav. 3 (1999), 17-24.

[2] W. A. Dudek, Y. B. Jun, and Z. Stojaković, On fuzzy ideals in BCC-algebras, Fuzzy Sets and Systems 123 (2001), no. 2, 251-258.

[3] Y. B. Jun and W. H. Shim, Fuzzy implicative hyper BCK-ideals of hyper BCK-algebras, Int. J. Math. Math. Sci. 29 (2002), no. 2, 63-70.

[4] Y. B. Jun and X. L. Xin, Fuzzy hyper BCK-ideals of hyper BCK-algebras, Sci. Math. Jpn. 53 (2001), no. 2, 353-360.

[5] Y. B. Jun, M. M. Zahedi, X. L. Xin, and R. A. Borzoei, On hyper BCK-algebras, Ital. J. Pure Appl. Math. 8 (2000), 127-136 . 
[6] F. Marty, Sur une generalization de la notion de groupe, 8th Congress Math. Scandinaves (Stockholm, 1934), pp. 45-49.

Jianming Zhan: Department of Mathematics, Hubei Institute for Nationalities, Hubei Province, Enshi 445000, China

E-mail address: zhanjianming@hotmai 1 .com

Dajing Xiang: Department of Mathematics, Hubei Institute for Nationalities, Hubei Province, Enshi 445000, China

E-mail address: xdj303@sohu.com

Zhisong Tan: Department of Mathematics, Hubei Institute for Nationalities, Hubei Province, Enshi 445000, China

E-mail address: es-tzs@263.net 


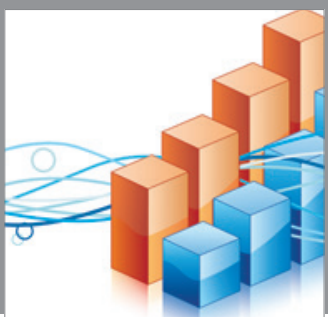

Advances in

Operations Research

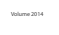

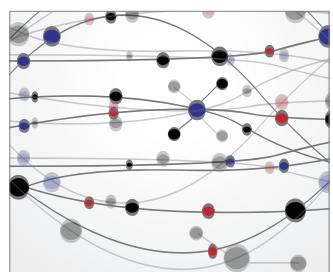

\section{The Scientific} World Journal
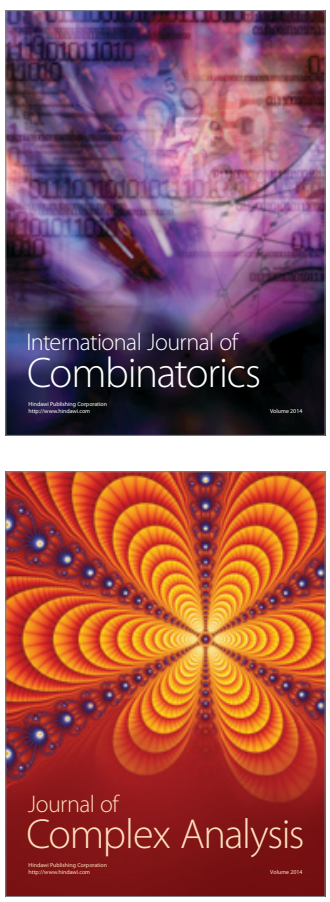

International Journal of

Mathematics and

Mathematical

Sciences
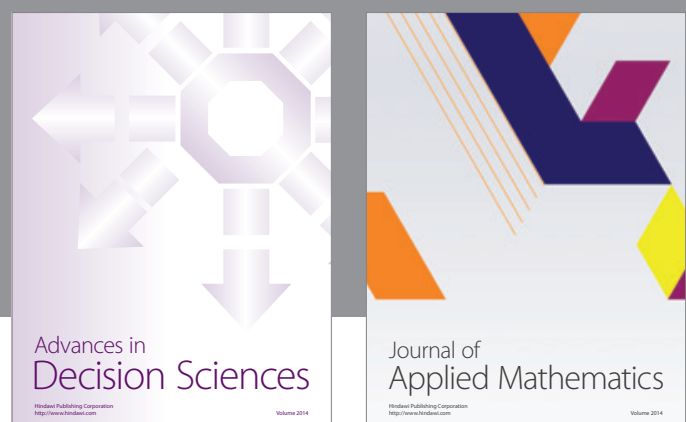

Journal of

Applied Mathematics
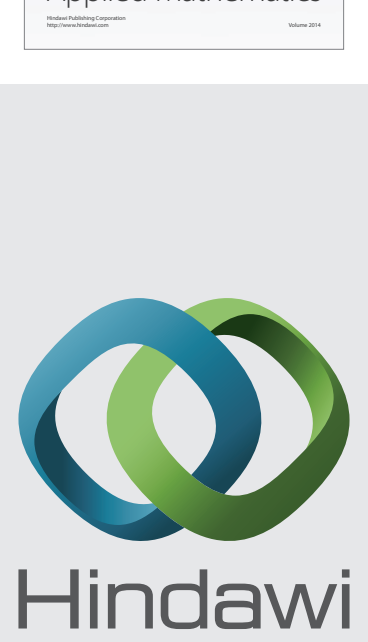

Submit your manuscripts at http://www.hindawi.com
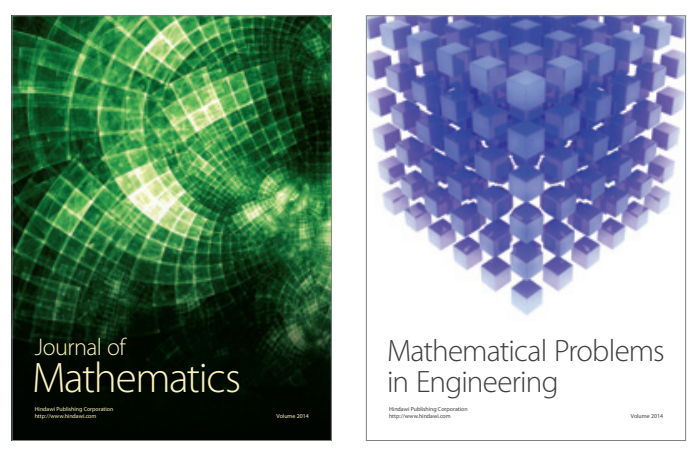

Mathematical Problems in Engineering
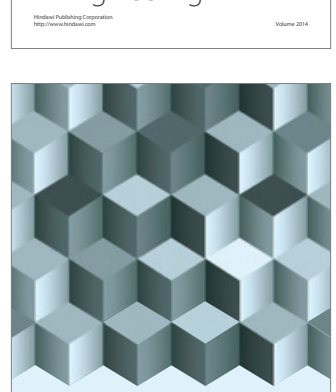

Journal of

Function Spaces
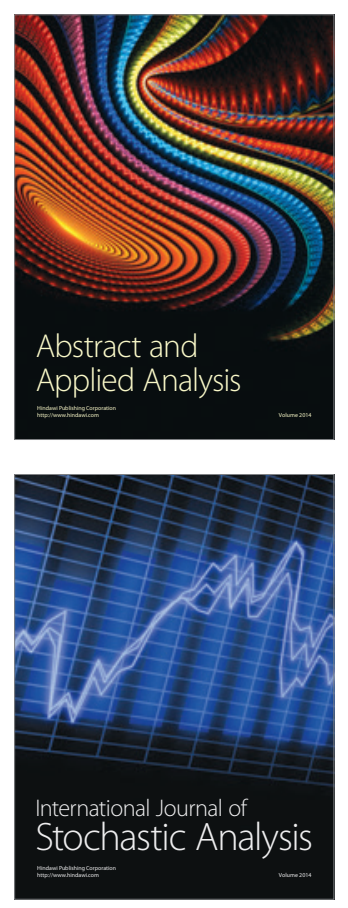

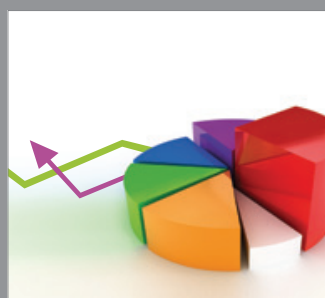

ournal of

Probability and Statistics

Promensencen
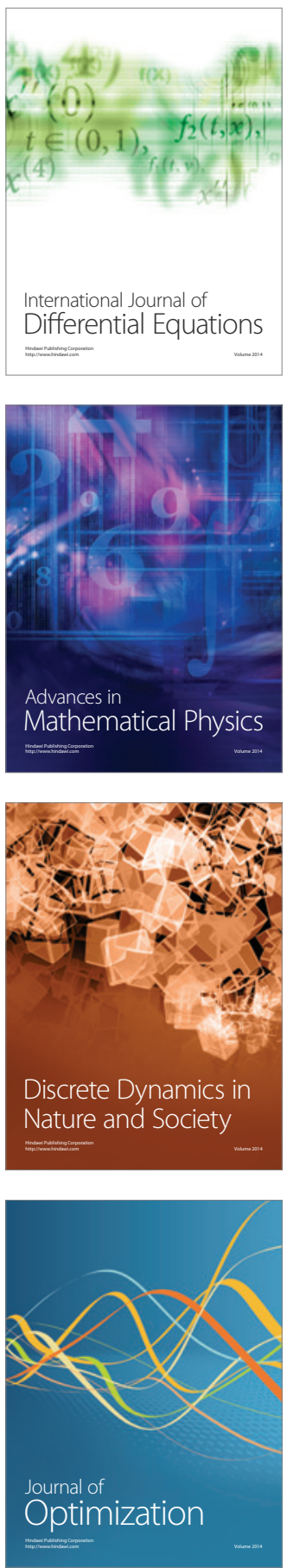